\title{
REFLECTANCE INFRARED SPECTROSCOPY ON OPERATING SURFACE ACOUSTIC WAVE CHEMICAL SENSORS DURING EXPOSURE TO GAS-PHASE ANALYTES
}

\author{
Ross C. Thomas, ${ }^{1}$ Andreas Hierlemann, ${ }^{2}$ Alan W. Staton, Mark Hill, and Antonio J. \\ $\operatorname{Ricco}^{3, *}$ \\ Microsensor Research \& Development Department, Sandia National Laboratories \\ Albuquerque, New Mexico 87185-1425, Phone: (505) 844-1405, Fax: (505) 844-1198, \\ e-mail: ajricco@sandia.gov
}

\begin{abstract}
We have developed instrumentation to enable the combination of surface acoustic wave (SAW) sensor measurements with direct, in-situ molecular spectroscopic measurements to understand the response of the SAW sensors with respect to the interfacial chemistry of surface-confined sensing films interacting with gas-phase analytes. Specifically, the instrumentation and software was developed to perform in-situ Fourier-transform infrared external-reflectance spectroscopy (FTIR-ERS) on operating SAW devices during dosing of their chemically modified surfaces with analytes. By probing the surface with IR spectroscopy during gas exposure, it is possible to understand in unprecedented detail the interaction processes between the sorptive SAW coatings and the gaseous analyte molecules. In this report, we provide details of this measurement system, and also demonstrate the utility of these combined measurements by characterizing the SAW and FTIR-ERS responses of organic thin-film sensor coatings interacting with gas-phase analytes.

\footnotetext{
*Author to whom correspondence should be addressed.

${ }^{1}$ Present address: Eltron Research, Inc., 5660 Airport Blvd., Boulder, Col., 80301

${ }^{2}$ Present address: Physical Electronics Laboratory, IQE, ETH Hoenggerberg HPT-H4.2, CH-8093 Zurich, Switzerland

${ }^{3}$ Present address: ACLARA Biosciences, Inc., 3906 Trust Way, Hayward, Calif., 94545

Prepared for publication as a Technical Note in Analytical Chemistry (11-98)
} 


\section{INTRODUCTION}

Chemical microsensors are widely used to monitor the presence of gas-phase organic analytes. ${ }^{1}$ Sensors that not only detect, but distinguish among a range of volatile organic compounds (VOCs) are needed for applications ranging from environmental cleanup and monitoring to contraband detection. As the demand for such sensor systems increases, it is necessary to develop novel sensing films with molecular specificity. $2-4,6$ In-situ molecular spectroscopic measurements on an operating chemical sensor device offer the potential to understand in detail the detection mechanisms of chemical recognition-based sensors.

Chemically functionalized surface acoustic wave (SAW) devices are commonly used to detect VOCs due to their extreme mass sensitivity to surface adsorbates. ${ }^{2-6}$ With our $97-\mathrm{MHz}$ SAW devices, a surface mass detection limit of ca. $100 \mathrm{pg} / \mathrm{cm}^{2}$ is routinely realized; higher frequency devices, coupled with carefully designed circuitry, can improve on this by two orders of magnitude or more. The exceptional sensitivity of these devices results from confinement of the acoustic energy near the device surface, coupled with frequency stability that allows measurement of SAW velocity changes with a resolution of 1 part in $10^{8} .2,7$

In many cases, organic monolayer or polymer thin films are immobilized on SAW devices to provide selectivity for chemical sensor applications. A detailed understanding of interfacial interactions between these chemically sensitive interfaces and key gas-phase analytes is helpful to tune the molecular specificity of the coatings. ${ }^{2-4,6}$ To this end, we have combined SAW measurements with direct, in-situ Fourier-transform infrared external-reflectance spectroscopy (FTIR-ERS). The FTIR-ERS measurements allow the study of changes in the IR signatures of sorption matrices and/or analyte molecules as the two interact. ${ }^{8-14}$

A schematic diagram of the experimental setup for the surface infrared measurements is shown in Figure 1. FTIR-ERS of organic thin films confined to Au substrates are sensitive to both the polarization state and angle of incidence of the infrared radiation. ${ }^{8-14}$ Using the polarization state dependence, it is possible to distinguish between IR absorbances in the nearsurface region and those of molecules in the gas phase. The sum of surface-parallel components 


\section{DISCLAIMER}

This report was prepared as an account of work sponsored by an agency of the United States Government. Neither the United States Government nor any agency thereof, nor any of their employees, make any warranty, express or implied, or assumes any legal liability or responsibility for the accuracy, completeness, or usefulness of any information, apparatus, product, or process disclosed, or represents that its use would not infringe privately owned rights. Reference herein to any specific commercial product, process, or service by trade name, trademark, manufacturer, or otherwise does not necessarily constitute or imply its endorsement, recommendation, or favoring by the United States Government or any agency thereof. The views and opinions of authors expressed herein do not necessarily state or reflect those of the United States Government or any agency thereof. 


\section{DISCLAIMER}

Portions of this document may be illegible in electronic image products. Images are produced from the best available original document. 
of the real and image (in the surface of the metal) electric-field vectors for $s$-polarized infrared radiation produces essentially no electric field at the Au surface at all angles of incidence, regardless of orientation of any molecular dipoles, which prevents IR absorption. In contrast, the incident and image electric-field vectors of $p$-polarized infrared radiation reinforce one another, this effect being maximized at grazing incidence. The $p$-polarized radiation with this enhanced surface-normal electric-field vector is absorbed only by those molecular dipoles that have a component perpendicular to the surface. ${ }^{8-14}$ Thus, surface-confined molecules and thin films with molecular dipoles having a surface-normal component appreciably absorb only $p$ polarized infrared radiation, while molecules in the gas phase absorb both polarizations equally. If the gas-phase conditions remain constant during the FTIR-ERS measurements, and using appropriate background corrections, it is possible to remove the contributions from gas-phase molecules by subtracting $s$ from $p$-polarized spectra, leaving only the spectrum of molecules and films in the near-surface region. This "selective absorption region" extends out from the surface to approximately one-quarter wavelength of the probing light, which results in a sampling region in the range of $0.6-5 \mu \mathrm{m} .15$

\section{EXPERIMENTAL DETAILS}

Thin-Film Sensor Coatings. To demonstrate the utility of the combined SAW/FTIR-ERS measurements, we coated custom-designed devices (shown schematically in Figure 1) with a variety of thin-film sensor coatings. In all cases, film deposition was on top of a 200-nm-thick $\mathrm{Au}$ thin film, thermally evaporated onto the active surface region of clean quartz SAW/FTIRERS device. A 20-nm-thick $\mathrm{Cr}$ layer was used to improve adhesion between the quartz and the Au film.

The first material studied was a polyisobutylene (PIB) thin film, deposited by spin coating from a dilute solution of the polymer in chloroform onto a SAW/FTIR-ERS device. The thickness of the PIB thin film depends on the solution concentration of the polymer and the speed of the spin coater. Since the sensitivity of the PIB coating to VOCs is related directly to 
film thickness, deposition conditions were chosen to provide readily measurable SAW and FTIR-ERS responses upon exposure to gas-phase tetrachloroethene.

We also prepared SAW/IR devices functionalized with a carboxylate-coordinated $\mathrm{Cu}^{2+}$ terminated self-assembled monolayer (SAM) surface. ${ }^{16-18}$ These composite films were prepared immediately upon removal of the SAW/FTIR-ERS devices from the Au evaporation system by (1) immersing the substrates in a $0.5 \mathrm{mM}$ ethanolic solution of 11mercaptoundecanoic acid (MUA; Aldrich, Milwaukee, WI) for $12 \mathrm{~h}$, (2) thoroughly rinsing with ethanol, deionized water, and ethanol again, (3) immersing these carboxylic acid-terminated monolayer films in a $2 \mathrm{mM}$ ethanolic solution of $\mathrm{Cu}\left(\mathrm{ClO}_{4}\right)_{2} \mathrm{O}_{6} \mathrm{H}_{2} \mathrm{O}$ (Aldrich) for $10 \mathrm{~min}$, and (4) thoroughly rinsing the composite monolayer film with ethanol before drying with $\mathrm{N}_{2}$. Previous FTIR-ERS and X-ray photoelectron spectroscopy measurements of similarly prepared composite monolayer films have confirmed the formation of the $(\mathrm{COO}-)_{2} / \mathrm{Cu}^{2+}$ interface. ${ }^{16-18}$

The last material studied was a modified cyclodextrin (3-O-butanoyl-2,6-di- $O$ - $n$-pentyl- $\gamma$ cyclodextrin, $C D$ ) a so-called cage compound, synthesized by the group of Prof. Schurig at the Institute of Organic Chemistry of the University of Tübingen, Germany; 19 a detailed description of the synthetic procedures can be found elsewhere. ${ }^{20}$ The CD was spray coated onto the devices using an airbrush. The response of the $\mathrm{CD}$-coated device was compared directly to that from two devices coated with conventional organic polymers. The polar polymer poly(etherurethane) (PEUT; Thermedics, Woburn, MA) and the nonpolar polyisobutylene (PIB, $\mathrm{MW} \approx 380,000 ;$ Aldrich) were used as representative conventional polymer coatings, nominally free of any molecular recognition moieties. The coatings of these polymers on the SAW/FTIRdevices were prepared by spin casting from chloroform solutions or spray coating from dichloromethane solutions; layer thicknesses ranged from $500 \mathrm{~nm}$ to $1 \mu \mathrm{m}$.

FTIR-ERS Measurements. Combined SAW/FTIR-ERS measurements were performed using a Nicolet Magna-IR 750 FTIR spectrometer, equipped with a custom-designed gas-flow test cell (Figure 1) that fits within a Seagull reflectance accessory (Harrick Scientific, Ossining, 
NY). The spectrometer utilizes a $\mathrm{KBr}$ beamsplitter and a liquid-nitrogen-cooled mercury cadmium telluride (MCT) detector. The function of the Seagull reflection accessory is to direct incident light from the spectrometer onto a reflective surface at selectable incident angles spanning the range $5^{\circ}$ to $87^{\circ}$ from surface normal, without the need for optical realignment; this accessory also collects the reflected light and directs it to the spectrometer's detector. The optical design results in no polarization dependence on the angle of incident radiation. For the present study, all spectra were acquired using infrared radiation incident on the substrate at $80^{\circ}$ from the surface normal, resulting in an elliptically shaped IR sampling area on the device surface approximately $30 \times 8 \mathrm{~mm}$.

The polarization of the infrared radiation was changed between $s$ and $p$ by rotating a wiregrid polarizer (International Crystal Labs, Garfield, NJ) $\pm 90^{\circ}$. This was accomplished with a custom-designed rotational stage that consists of two components, one that holds the polarizer and another that fits onto the Seagull reflection accessory. The polarizer holder was configured to rotate exactly $\pm 90^{\circ}$ by moving a lever that extends away from this component and through the front of the spectrometer sample compartment. A Bimba double-acting air cylinder (Bimba, Monee, IL) having a 2 in stroke and a solenoid-operated valve were used to rotate the polarizer under computer control.

The following protocol was used to acquire FTIR-ERS single-beam spectra: (1) the test-cell was purged with $\mathrm{N}_{2}$, (2) an s-polarized background spectrum was obtained, (3) the polarizer was rotated $+90^{\circ}$, (4) a $p$-polarized background spectrum was obtained, (5) the SAW/FTIR-ERS device was exposed to a target analyte, (6) a p-polarized sample spectrum was obtained during analyte exposure, (7) the polarizer was rotated $-90^{\circ},(8)$ an $s$-polarized sample spectrum was obtained, and (9) the polarizer was rotated $+90^{\circ}$. Additional scans were taken by repeating steps 5 through 9. These steps could all be performed under (automated) software control. Nicolet provides the libraries necessary to operate the spectrometer in a Visual Basic (Microsoft Corp.) environment, allowing the compilation of a program to fully control the rotation of the polarizer in sequence with the FTIR-ERS scans and SAW measurements. The polarizer was actuated 
using the RS-232 port of the computer to send a "hi/lo" signal to the solenoid-operated valve; an optical isolator switch between these two components protected the computer from electrical noise.

The infrared response of the gas-phase molecules in the gas-flow test cell was determined using Eq. 1:

$$
A_{\operatorname{gas}}(\lambda)=\log \left(S_{S}(\lambda) B_{S}(\lambda)\right)
$$

where $S$ and $B$ refer to the sample and background intensities, respectively, and the subscript $S$ indicates that the data were acquired with $s$-polarized infrared radiation (which is transparent to near-surface species). Information about the infrared response of surface interactions was determined using Eq. 2:

$$
A_{\text {surface }}(\lambda)=\log \left(S_{p}(\lambda) / B_{p}(\lambda)\right)-\log \left(S_{S}(\lambda) / B_{S}(\lambda)\right)
$$

where the subscript $p$ indicates that the data were acquired with $p$-polarized infrared radiation. No baseline correction to the spectra was necessary as long as each set of $p$ - and $s$-polarized scans was acquired under the same conditions within a short time period, typically $30-60 \mathrm{~s}$. The application of Eq. 2 enables the study of surface interactions between SAW sensor coatings and gas-phase analytes by eliminating the infrared absorbances that arise from gaseous analyte present in the test cell.

SAW/FTIR-ERS Flow Cell. The flow cell consists of two stainless-steel body components held together by four 6-32 stainless-steel screws; a Viton ${ }^{\mathrm{TM}} \mathrm{O}-$-ring compressed between the two sections provides a gas-tight seal. The cover section has six SMB-type high-frequency connectors for three independent SAW delay line measurements, four O-ring sealed window ports, equipped with $\mathrm{KBr}$ windows, for $45^{\circ}$ - and $80^{\circ}$-from-normal spectral measurements, and two gas-flow ports equipped with $1 / 8$ inch Swagelok ${ }^{\circledR}$ fittings. Spring-loaded Au or Rh tipped 
"pogo" pins (Newark Electronics) located at the bottom of this component make low-resistance, noise-free contacts with the Au bond pads of the SAW delay lines. The base section contains a recessed region to locate the SAW/FTIR-ERS device, as well as two liquid-flow ports, equipped with $1 / 8$ inch Swagelok $®$ fittings, used to maintain the fixture at a constant temperature; stability was $\pm 0.1^{\circ} \mathrm{C}$.

SAW Devices and Measurements. SAW measurements were accomplished using 97$\mathrm{MHz}$, ST-quartz devices designed and fabricated at Sandia National Laboratories using UV lithography and physical vapor deposition methods. ${ }^{21}$ Five sets of independent interdigital $\mathrm{Au}$ transducers, $200 \mathrm{~nm}$ thick with a $50 \mathrm{~nm} \mathrm{Cr}$ or Ti adhesion layer, were defined on a $41 \times 15.5$ $\mathrm{mm} \mathrm{ST-quartz} \mathrm{substrate} \mathrm{(Figure} \mathrm{1).} \mathrm{It} \mathrm{was} \mathrm{necessary} \mathrm{to} \mathrm{increase} \mathrm{the} \mathrm{overall} \mathrm{dimensions} \mathrm{of} \mathrm{the}$ SAW sensor compared to devices typically used in our laboratory to accommodate the FTIRERS measurements. Only the three center delay lines were contacted by the pogo pins and used for SAW measurements. Each transducer consists of 50 finger pairs with a periodicity of $32 \mu \mathrm{m}$ (individual fingers are $8 \mu \mathrm{m}$ wide and $1.7 \mathrm{~mm}$ long). Center-to-center separation of input and output transducers is $10 \mathrm{~mm}$; parallel adjacent delay lines are separated by $6.5 \mathrm{~mm}$. A detailed frequency analysis of the operating characteristics of these devices revealed that the "cross talk" between the different delay lines was negligible. The response of three independent SAW . devices, therefore, could be measured simultaneously for each experiment.

The SAW measurements were performed according to previously described procedures. $7,17,22$ Approximately $30 \mathrm{~min}$ were required to stabilize the temperature of the SAW/FTIR-ERS devices under ultrahigh purity $\mathrm{N}_{2}$ before taking the first measurements. The chemically modified SAW/FTIR-ERS devices were exposed to concentration steps of selected gas-phase analytes in a $\mathrm{N}_{2}$ carrier gas stream using a computer-controlled flow system. ${ }^{23}$

Surface acoustic waves are excited and detected by input and output interdigital transducers formed on a piezoelectric quartz substrate. ${ }^{2}$ An alternating voltage applied to the input transducer launches a Raleigh wave that traverses the crystal surface; the output transducer 
converts the wave back into an electrical signal. If the SAW velocity is perturbed only by mass loading variations, the change in frequency, $\Delta f$, is related to the change in adsorbed mass per area, $\Delta(m / A)$, by Eq. $3: 2,3,21$

$$
\frac{\Delta f}{f_{0}}=-\kappa c_{m} f_{0} \cdot \Delta(m / A)
$$

where $c_{m}$ is the mass sensitivity $\left(1.33 \mathrm{~cm}^{2} / \mathrm{g}-\mathrm{MHz}\right.$ for ST-quartz), $f_{0}$ is the unperturbed oscillator frequency $(97 \mathrm{MHz})$, and $\kappa$ is the fraction of the center-to-center distance between the transducers covered by the chemically sensitive film. In addition to mass changes, the attenuation of the surface acoustic wave, $\alpha$, can be related to changes in the viscoelastic properties of surface-confined adsorbates. 2,21 It is typically normalized by division by the wavenumber, $k$. Changes in the dimensionless quantity $\alpha / k$ (simply referred to as "attenuation") can then be compared in magnitude to concurrent changes in $\Delta f f_{0}$ to estimate their relative significance in a qualitative sense. 2,24

The response of a film-coated SAW device to vapor-phase analytes depends upon many parameters. Sorption-related increases in film mass lead to decreases in wave velocity, and hence oscillation frequency. Changes in viscoelastic properties of the film also affect the wave velocity, as well as the amplitude. Other parameters such as film electrical conductivity and permittivity can affect both SAW velocity and amplitude, but only for ranges of these parameters that do not apply to the experiments described here. It is important to note that acoustically thin polymer films must be used in order to interpret SAW response solely in terms of mass loading.2,24 An elastic film, generally including metals, ceramics, and many glassy polymers, is considered acoustically thin under the following conditions: 


$$
h \ll \Lambda_{0} \times\left(\frac{v_{f}}{v_{0}}\right)^{2}
$$

where $h$ is the film thickness, $\Lambda_{0}$ is the SAW wavelength, and $v_{0}$ is the SAW velocity, and $v_{f}$ is the acoustic shear-wave velocity in the film (derivation based on ref. 24). If $h$ is no more than 1 - $3 \%$ of the product $\Lambda_{0}\left(v_{f} / v_{0}\right)^{2}$, then the film is likely to behave as "acoustically thin". A detailed discussion of acoustically thick versus thin films can be found in a paper by Martin et al. 24

\section{RESULTS AND DISCUSSION}

We illustrate the utility of simultaneous SAW/FTIR-ERS measurements with three different examples. The first demonstrates the monitoring of the gas-phase concentrations of an analyte, in addition to the determination of the amount of analyte sorbed into the coating matrix (partitioning) in the case of nonspecific (physical) sorption. The second shows the sensitivity of the method to a single organic monolayer on the SAW device. In the third example, we demonstrate how the SAW/FTIR-ERS method can be used to detect spectral changes upon analyte absorption due to specific chemical interactions between coating material and analyte molecules in the gas phase, in the context of "molecular recognition" of the analyte.

Partitioning. Figure 2 shows the SAW response of a PIB-coated SAW/FTIR-ERS device exposed to step concentrations of vapor-phase tetrachloroethene $\left(p / p_{s a t}=10,15\right.$, and $20 \%$ at $293 \mathrm{~K}$ ). The device was purged with dry $\mathrm{N}_{2}$ for $3 \mathrm{~min}$ before and after each 3-min exposure. The negative frequency shifts indicate a mass increase; as expected, the frequency shift increases monotonically with the concentration of $\mathrm{C}_{2} \mathrm{Cl}_{4}$, ranging from 85 to $200 \mathrm{ppm}$ of 97 $\mathrm{MHz}(8.25-19.4 \mathrm{kHz})$. The baseline is stable and the sensor signals reproducible. For each exposure, the IR spectra were recorded as soon as the SAW response stabilized, indicating equilibrium. The sensitivity of the device is related to the thickness of the PIB coating: a thicker 
coating gives a larger frequency shift, but the time to reach equilibrium increases. We have not attempted to optimize the response characteristics (i.e., to balance sensitivity against response time) of the thin-film coatings for the experiments discussed here.

The SAW response data were compared directly to the response of a "standard" SAW device, ${ }^{21}$ which had the same nominal thin-film coating and thickness, by connecting the gas test fixtures for both SAW and SAW/FTIR-ERS in series. The reproducibility of the frequency shifts between the two SAW device types was approximately $\pm 10 \%$, using the average of the three SAW responses provided by the SAW/FTIR substrate. The variation among the three individual sensors located on a single SAW/FTIR-ERS substrate was larger, about $20 \%$. It is reasonable to expect such discrepancies as a result of variations in the thickness of the spraycoated PIB thin film over the rather large device used for the SAW/FTIR measurements.

Because of the locations of various system components, a 5-m length of 1/4-in stainlesssteel tubing connected the gas/vapor manifold with the spectroscopic unit. To overcome long equilibration and purging times, we used a rather high flow rate, $1 \mathrm{~L} / \mathrm{min}$. It could prove beneficial to shorten considerably the gas paths, by moving spectrometer and manifold closer, to be able to use lower flow rates.

Figure 3 shows $p$-polarized FTIR-ERS spectra of tetrachloroethene molecules in the gasflow test cell, as well as $(p-s)$ difference spectra, demonstrating the enrichment of the analyte within the PIB thin film. The IR signatures displayed in Fig. 3A are completely identical to those of the pure gaseous analyte obtained without a surface reflection apparatus. Spectra were acquired as 100 signal-averaged scans at $1 \mathrm{~cm}^{-1}$ resolution, resulting in a measurement time of approximately $150 \mathrm{~s}$. The Fourier transformation was performed with boxcar apodization. These data were acquired after the frequency shifts shown in Figure 2 reached steady state. The background spectrum was obtained under a pure nitrogen purge prior to exposing the SAW/FTIR-ERS device to the analyte.

In Figure 3A, there are three main peaks. The peak with the highest intensity, at $915 \mathrm{~cm}^{-1}$, can be assigned to the $-\mathrm{CCl}_{2}$ symmetric stretching. The peak at $782 \mathrm{~cm}^{-1}$ can be assigned to 
the $-\mathrm{CCl}_{2}$ asymmetric stretching. The peak with the lowest intensity probably results from combinations of the fundamental modes. ${ }^{25}$

In analogy to the SAW measurements, the intensity of the infrared bands monotonically increases with $\mathrm{C}_{2} \mathrm{Cl}_{4}$ concentration. It is thus logical to compare directly the $\mathrm{SAW}$ response from Figure 2 with the peak intensity of the band at $915 \mathrm{~cm}^{-1}$ in Figure 3A. Before making such a comparison, however, it is necessary to separate contributions to the IR absorbance from analyte sorbed in the film-to which the SAW responds-from those associated with the analyte in the gas phase.

The $p$-polarized spectra include large contributions from gas-phase analyte, added to a significantly smaller contribution from the analyte molecules sorbed in the PIB matrix. Although the enrichment factor (or partition coefficient) of $\mathrm{C}_{2} \mathrm{Cl}_{4}$ in PIB is in the range of 2000 , plus a small absorbance enhancement due to the additive effect of the image electric-field vector (in the Au film), the optical path in the PIB matrix (approx. $2 \mu \mathrm{m}$ ) is over $10^{4}$ times shorter than in the gas phase (approx. $6 \mathrm{~cm}$ ). We point out that a very carefully designed fixture could bring the window material much closer to the reflective Au surface, diminishing the path length in the gas phase by an order of magnitude or more. No spectral changes (relative intensity, peak position) are recorded as a consequence of sorption of the $\mathrm{C}_{2} \mathrm{Cl}_{4}$ in the PIB, indicating that this sorptive process is purely physical.

The surface selection rule states that $s$-polarized infrared radiation is not absorbed by nearsurface molecular dipoles of any orientation. ${ }^{8-14}$ Measurements using s-polarized IR light therefore provide an excellent method to reliably verify the concentration of the vapor-phase analyte to which the chemical microsensors are exposed. Figure 3B shows difference spectra of $\mathrm{C}_{2} \mathrm{Cl}_{4}$ sorbed into PIB, obtained by subtracting $s$ - from $p$-polarized spectra. This nominally eliminates gas-phase contributions, leaving only the spectrum of sorbed analyte, allowing us to assess the enrichment factor or partition coefficient spectroscopically. Again, the intensity of the difference infrared peaks increases linearly with gas-phase concentration. 
The measurements described here are analogous to polarization-modulation (PM) FTIRERS experiments, from which it is possible to obtain both reference and PM data simultaneously. $13,14,18,26-28$ The synchronous detection of the PM-FTIR method is more ideally suited to detect the relatively small difference between $p$ - and $s$-polarized spectra when the concentration of analyte in the gas phase is significant, and is more immune to experimental drift, which can degrade spectral quality. Several reports in the literature illustrate the usefulness of PM-FTIR measurements to understand complex interactions of thin films and monolayers at metal surfaces. ${ }^{18,26-28}$ The most common configuration uses a photoelastic modulator with conventional electronics that utilize a lock-in-amplifier to obtain real-time surface infrared information, and typically requires a fairly expensive, dedicated setup. In comparison, the experimental setup for our FTIR-ERS measurements only requires the ability to rotate a standard polarizer $\pm 90^{\circ}$ to acquire both $p$ - and s-polarized spectra. Although this simple method does not achieve all of the benefits gained with real-time PM FTIR-ERS measurements, it is considerably less expensive to implement and can provide detailed information about the interaction of gas-phase molecules with a chemically sensitive interface. ${ }^{4}$

Monolayers. The SAW and FTIR-ERS data shown in Figures 4A and 4B for a $\mathrm{Cu}^{2+} / \mathrm{MUA}-$ functionalized SAW device demonstrate the sensitivity of our measurements, which provide a basis to study interactions between composite monolayer films and gas-phase adsorbates. ${ }^{16-18}$ Although our SAW measurements are sensitive to approximately $100 \mathrm{pg} / \mathrm{cm}^{2}$, typical absorbances for organic monolayer films are on the order of $10^{-3}$, and therefore FTIR-ERS requires a high degree of instrumental sensitivity and stability as well as measurement repeatability. ${ }^{8}$ The measured absorbance intensity may diminish further under conditions that enlarge the focal beam and reduce the spectral resolution. A typical signal-to-noise ratio in FTIR-ERS measurements is comparable to that of transmission infrared analysis. ${ }^{8}$

The SAW measurements (Figure 4A) were performed according to the following protocol. Initially, the MUA/Cu ${ }^{2+} \mathrm{SAM}$-coated device was exposed to pure $\mathrm{N}_{2}$ between 0 and $8 \mathrm{~min}$. 
These data illustrate the stability of the SAW measurements. Next, the device was exposed to diisopropylmethylphosphonate (DIMP) at $25 \%$ of its saturation vapor pressure at $293 \mathrm{~K}\left(p / p_{\text {sat }}\right.$ $=0.25$ ) between 8 and $20 \mathrm{~min}$. The magnitude of the frequency shift is consistent with the adsorption of approximately 2 layers of DIMP on top of the coordinatively unsaturated $\mathrm{Cu}^{2+}$, assuming the layers have the density of the bulk liquid and that the SAW response is entirely due to mass loading. ${ }^{16-18}$ Finally, the device was exposed to DIMP at $p / p_{s a t}=0.5(293 \mathrm{~K})$ between 20 and $30 \mathrm{~min}$, yielding a frequency shift consistent with approximately 4 layers of adsorbed DIMP: the SAW response is approximately linear over this concentration range. It is noteworthy that previous research studies have shown it is possible to dramatically influence the extent of multilayer formation by changing either the solution-phase formation time of the monolayer or the grain size of the Au surface supporting the composite monolayer. ${ }^{17}$

The FTIR-ERS measurements (Figure 4B) were performed at approximately 6,18 , and 28 min with respect to the SAW measurements (Figure 4A). These data were acquired as 128 signal-averaged scans at 4- $\mathrm{cm}^{-1}$ resolution, resulting in a measurement time of approximately 60 s. The Fourier transformation was performed with boxcar apodization. The $p-s$ difference spectra of Figure 4B effectively eliminate the spectral contribution from the gas-phase analyte. The initial spectrum shows the instrumental baseline to illustrate the sensitivity and linearity of the detector response. The other two spectra correspond to exposure of the SAW/FTIR-ERS device exposed to DIMP at $p / p_{s a t}=0.25$ and 0.5 . Several infrared absorption peaks characteristic of DIMP are present: the $\mathrm{P}-\mathrm{O}$ stretch at $1016 \mathrm{~cm}^{-1}$, the hydrogen-bonded $\mathrm{P}=\mathrm{O}$ stretch at $1206 \mathrm{~cm}^{-1}$, the resonance-split symmetric deformation of the P-bound $\mathrm{CH}_{3}$ groups at $1315 \mathrm{~cm}^{-1}$, and the asymmetric $\mathrm{C}-\mathrm{H}$ stretch of the isopropyl methyl groups centered at 2984 $\mathrm{cm}^{-1}$. These results are in excellent agreement with previously published studies detailing the interactions of this $\mathrm{Cu}^{2+}$-terminated monolayer with DIMP. ${ }^{8,16-18}$ The magnitudes of the peaks all increase linearly with DIMP concentration. 
Molecular recognition. Molecular recognition is an important topic when searching for new, selective coating materials for chemical sensing. ${ }^{4}$ In the case of the cyclodextrin (CD) on which we report here, oriented adsorption of the molecules within the $C D$ torus (inclusion of the analyte molecule within the supramolecular cage) seems to control the recognition process. Figures 5A and 5B show the SAW and FTIR-ERS responses, respectively, of cyclodextrin thin films during exposure to R-methyl lactate.

The frequency shifts of the CD-coated SAW/FTIR-ERS devices exposed to $1 \% \mathrm{R}$-methyl lactate in nitrogen carrier gas (at $303 \mathrm{~K}$ ) were approximately $2 \mathrm{ppm}$ of $97 \mathrm{MHz}$ (Figure 5A). The equilibration times are relatively short, despite a total flow volume of $1 \mathrm{~L}$ due to the long tubing connection between the gas manifold and the FTIR spectrometer. The short-term frequency noise of the large IR-SAW device is between 3 and $5 \mathrm{~Hz}$ (RMS), slightly higher than for the single $97 \mathrm{MHz}$ SAW devices. The SAW response was quite reproducible.

Figure 5B shows the relevant portions of the IR-spectra ( $p$ polarization only) of methyl lactate recorded simultaneously with the SAW response. In this case, the spectral changes are most easily observed simply by examining the $p$ spectra, which include contributions from both the gas phase and the near-surface region, with a background spectrum of the thin-film coating under nitrogen purge (in the absence of analyte) subtracted out. We then compare the features of spectra obtained for the sorption of methyl lactate by ordinary organic polymers with the results for sorption by the cyclodextrin. From bottom to the top, the absorbance spectra shown are for R-methyl lactate in PIB, PEUT ( $p / p_{s a t}=5 \%$ at $\left.293 \mathrm{~K}\right)$, and R-methyl lactate $\left(p / p_{\text {sat }}=\right.$ $5 \%$ and $10 \%$ at $293 \mathrm{~K}$ ) in the $\mathrm{CD}$ film. Spectra were acquired as 100 signal-averaged scans at $1 \mathrm{~cm}^{-1}$ resolution, resulting in a measurement time of approximately $150 \mathrm{~s}$. The Fourier transformation was performed with boxcar apodization. The data were acquired after the frequency changes shown in Figure 5A reached equilibrium. These spectra represent the raw data and are not processed in any way.

The spectra of R-methyl lactate in PIB and PEUT are identical to the gas-phase spectra of this analyte, since only physisorption is involved here. The main change in the spectrum of 
methyl lactate sorbed into the $\mathrm{CD}$, in comparison to the spectra for sorption into PEUT or PIB, is a new peak in the $\mathrm{OH}$ region, indicating a strong, specific interaction between the methyl lactate and the cyclodextrin via intermolecular hydrogen bonds. The peak maximum is at 3468 $\mathrm{cm}^{-1}$; the "regular", non-H-bonded $\mathrm{OH}$ peak is at $3575 \mathrm{~cm}^{-1}$. A feature of the spectral change is saturation of the magnitude of this effect at relatively low analyte concentration: the new ( $\mathrm{H}-$ bond) $\mathrm{OH}$ peak of methyl lactate nearly attains its maximum intensity at $p / p_{\text {sat }}=5 \%(293 \mathrm{~K})$. Increasing the analyte concentration to $p / p_{\text {sat }}=10 \%$ (top spectrum) or $20 \%$ (not shown) results in a large increase in intensity for the "regular" $\mathrm{OH}$ peak at $3575 \mathrm{~cm}^{-1}$, which is associated principally with the methyl lactate free in the gas phase, but causes little increase in the Hbonding shoulder at $3468 \mathrm{~cm}^{-1}$, which is associated entirely with sorbed methyl lactate. This saturation phenomenon is to be expected: the number of preferential sorption sites (the $\mathrm{CD}$ tori) is limited, and once they are all occupied-near $p / p_{s a t}=5 \%$ in this case-the IR absorbance associated with binding in these sites increases no further.

Our SAW/FTIR results are consistent with conclusions derived earlier from TSMR data. 19 In most cases where specific chemical interactions are expected, we record distinctive changes in the IR spectra, together with anomalously large SAW sensor responses, upon analyte dosing. In contrast, for standard physisorption processes (i.e., the conventional polymers PEUT or PIB interacting with various volatile organics), no changes in the IR spectra occur and the SAW responses are much smaller. For a detailed SAW/FTIR study of molecular recognition, see ref. 4.

\section{SUMMARY and CONCLUSIONS}

The method of simultaneous SAW/in-situ FTIR-ERS measurements is an effective, powerful tool for elucidating interactions between coatings and gaseous analytes and provides a basis to understand the operating characteristics of the SAW sensor with respect to the interfacial chemistry of surface-confined sensing films interacting with gas-phase analytes. The gas-phase concentration of the analyte and, importantly, the enrichment or partitioning in the coating 
material, can be determined independently of simultaneous SAW measurements for standard physisorption processes, as demonstrated using polyisobutylene films. Due to the high sensitivity of the FTIR-ERS measurements, we were able to monitor quantitatively (SAW) and qualitatively (spectroscopic information) the interfacial interactions during the exposure of monolayers to vapor-phase molecules. Most important, the method of combining SAW with insitu FTIR-ERS experiments enables us to provide direct evidence for chemically specific interactions between volatile analytes and coating materials on acoustic wave gas sensors in the context of molecular recognition. Whénever specific chemical interactions (inclusion into a cage compound in this case) are present, we record distinctive changes in the IR spectra, together with anomalously large SAW sensor responses, upon analyte dosing. In contrast, for standard physisorption processes, as exemplified by the conventional polymers PEUT and PIB, no changes in the IR spectra occur and the SAW responses are smaller.

As the demand for SAW chemical sensor systems increases, it is necessary to develop novel sensing films having a high level of molecular specificity. Our combined SAW and FTIR-ERS measurements fully illustrate a technically sound approach to test the sensitivity, selectivity, and reliability of organic thin-film interfaces used in such applications.

\section{ACKNOWLEDGMENTS}

We gratefully acknowledge several discussions with Richard M. Crooks (Texas A\&M University) that led to the concept of the integrated SAW/FTIR measurement system. We thank Gerald Fowler (Sandia, retired) for his excellent design work developing the polarizer rotational stage used in these experiments, and the competent technical assistance of Mary-Anne Mitchell (Sandia). The authors also acknowledge Li Sun and Richard M. Crooks (Texas A\&M University) for assistance with spectral interpretations. Finally, we thank Prof. V. Schurig (Institute of Organic Chemistry, University of Tübingen, Germany) for providing the modified cyclodextrin. Sandia is a multiprogram laboratory operated by Sandia Corporation, a Lockheed 
Martin Company, for the United States Department of Energy under Contract DE-AC0494AL85000. 


\section{REFERENCES}

1. Hughes, R. C.; Ricco, A. J.; Butler, M. A.; Martin, S. J. Science 1991, 254, 74.

2. Ballantine, D. S.; White, R. M.; Martin, S. J.; Ricco, A. J.; Frye, G. C.; Zellers, E. T.; Wohltjen, H. Acoustic Wave Sensors: Theory, Design, and Physico-Chemical Applications; Academic Press: San Diego, 1997.

3. Grate, J. W.; Martin, S. J.; White, R. M. Anal. Chem. 1993, 65, 940A.

4. Hierlemann, A.; Ricco, A. J.; Bodenhöfer, K.; Göpel, W. Anal. Chem. submitted for publication.

5. Ward, M. D.; Buttry, D. A. Science 1990, 249, 1000.

6. Ricco, A. J.; Crooks, R. M.; Osbourn, G. C. Acc. Chem. Res.. 1998,31, 289.

7. Ricco, A. J.; Frye, G. C.; Martin, S. J. Langmuir 1989, 5, 273.

8. Crooks, R. M.; Xu, C.; Sun, L.; Hill, S. L.; Ricco, A. J. Spectroscopy 1993, 8, 28.

9. Porter, M. D. Anal. Chem. 1988, 60, 1143 A.

10. Bubeck, C.; Holtkamp, D. Adv. Mater. 1991, 3, 32.

11. Greenler, R. G. J. Vac. Sci. Technol. 1975, 12, 1410.

12. Greenler, R. G. J. Chem. Phys. 1966, 44, 310.

13. Ferraro, J. R.; Basile, L. J. Fourier transform Infrared Spectroscopy; Academic Press: New York, 1985; chapters 7-8.

14. Yates, Jr., J. T.; Madey, T. E. Vibrational Spectroscopy of Molecules on Surfaces; Plenum Press: New York, 1987; chapter 7.

15. Corn, R. Private communication.

16. Kepley, L. J.; Crooks, R. M. Anal. Chem. 1992, 64, 3191.

17. Thomas, R. C.; Sun, L.; Crooks, R. M.; Ricco, A. J. Langmuir 1991, 7, 620.

18. Crooks, R.M.; Yang, H. C.; McEllistrem, L. J.; Thomas, R. C; Ricco, A. J. Faraday Discuss. 1997,107, 285. 
19. Bodenhöfer, K.; Hierlemann, A.; Juza, M.; Schurig, V.; Göpel, W. Anal. Chem., 1997, 69, 4017.

20. König, W. A.; Krebber, R.; Mischnick, P. J. High Resolut. Chromatogr. 1989, 11, 732.

21. Ricco, A. J.; Martin, S. J. Thin Solid Films 1992, 206, 94.

22. Sun, L.; Thomas, R. C.; Crooks, R. M.; Ricco, A. J. J. Am. Chem. Soc. 1991, 113, 8550.

23. Martin, S. J.; Ricco, A. J.; Ginley, D. S.; Zipperian, T. E. IEEE Trans. on UFFC 1987, $U F F C-34,142$.

24. Martin, S. J.; Frye, G. C.; Senturia, S. D. Anal. Chem. 1994, 66, 2201.

25. Bernstein, H. J. J. Chem. Phys. 18, 478 (1950), and Pouchert, C. J. Aldrich Library of FTIR-spectra Vol. 3, 1989, Milwaukee, WI.

26. Barner, B. J.; Green, M. J.; Saez, E. I.; Corn, R. M. Anal. Chem. 1991, 63, 55.

27. Richmond, W. N.; Faguy, P. W. Anal. Chem. 1996, 68, 621.

28. Green, M. J.; Barner, B. J.; Corn, R. M. Rev. Sci. Instrum. 1991, 62, 1426. 


\section{FIGURE CAPTIONS}

Figure 1. Test fixture and optical setup used to perform simultaneous SAW/in-situ FTIR external reflectance measurements. The source and detector $(\mathrm{MCT}=$ mercurycadmium telluride) are part of a commercial FTIR instrument, the polarizer is a manually rotated wire grid type, and a "seagull" reflection accessory (not shown) is used to provide and collect light at an angle of about $80^{\circ}$ off normal. Three of the five SAW delay lines (ST-cut quartz; $97 \mathrm{MHz}$ ) are electrically connected using the high-frequency $(\mathrm{HF})$ connectors to make multiple measurements of coating/analyte interactions occurring in the central region of the device; the selective coating is applied on top of the reflective Au layer, which is $40 \times 8 \mathrm{~mm}$ in area.

Figure 2. Frequency changes of a PIB-coated SAW/FTIR-ERS device exposed to step concentrations $\left(p / p_{\text {sat }}=10,15,20 \%\right.$ at $\left.293 \mathrm{~K}\right)$ of gas-phase $\mathrm{C}_{2} \mathrm{Cl}_{4}$. The magnitude of the negative frequency shifts increases linearly with the analyte concentration in the gas phase.

Figure 3. (A) FTIR-ERS spectra of $\mathrm{C}_{2} \mathrm{Cl}_{4}$ in the presence of a PIB matrix on the FTIR/SAW device. The $p$-polarized data include contributions from analyte molecules in both the gas phase and sorbed into the PIB matrix. The FTIR spectra were collected after the SAW responses shown in Figure 2 reached equilibrium.

(B) FTIR-ERS difference spectra of $\mathrm{C}_{2} \mathrm{Cl}_{4}$ sorbed in PIB. Subtraction of $s$ - from $p$-polarized data yields the signal associated only with the analyte molecules absorbed in the PIB matrix (partitioning).

Figure 4. (A) SAW and (B) FTIR-ERS responses for DIMP adsorbed to a carboxylatecoordinated $\mathrm{Cu}^{2+}$-terminated monolayer surface. These combined measurements 
provide information about the interfacial interactions between the composite monolayer and the adsorbed analyte.

Figure 5. SAW and FTIR-ERS responses to the sorption of R-methyl lactate by a modified cyclodextrin. (A) SAW response for $p / p_{\text {sat }}=1 \%$ at $303 \mathrm{~K}$; (B) FTIR-ERS data for $p / p_{\text {sat }}=10 \%$ and $5 \%$ at $293 \mathrm{~K}$ (labeled CD 10\%, CD 5\%). Also included for comparison in (B) are the spectra of R-methyl lactate sorbed by the conventional polymers PIB and PEUT. The appearance of a new band in both CD spectra at 3468 $\mathrm{cm}^{-1}$, a consequence of hydrogen bond formation between analyte and coating, establishes the presence of a specific analyte/matrix interaction. For PIB and PEUT, the FTIR spectra are identical to those of the free analyte in the gas phase, indicating a total absence of specific chemical interaction. 


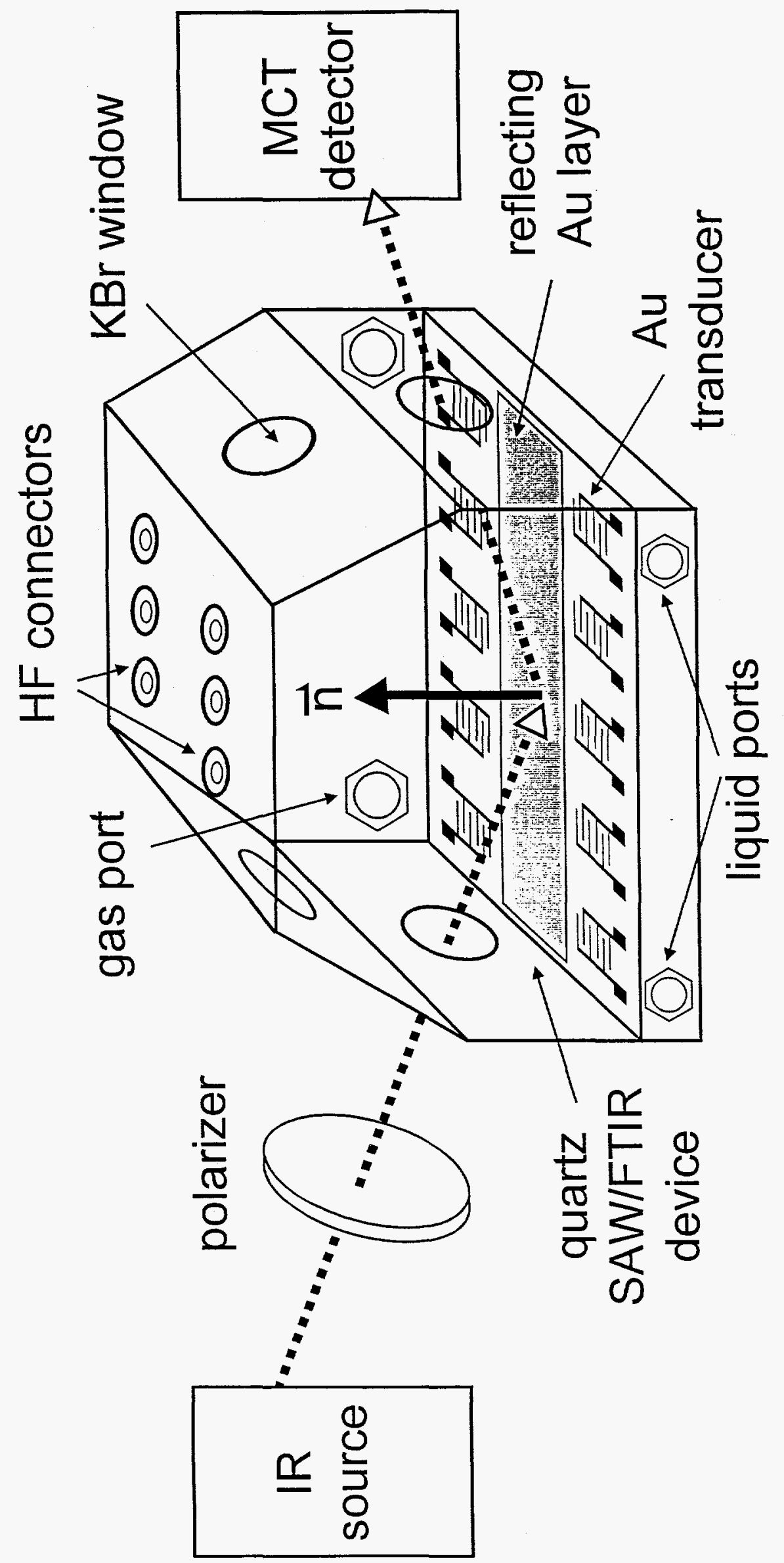

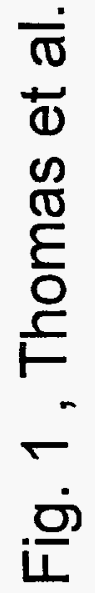




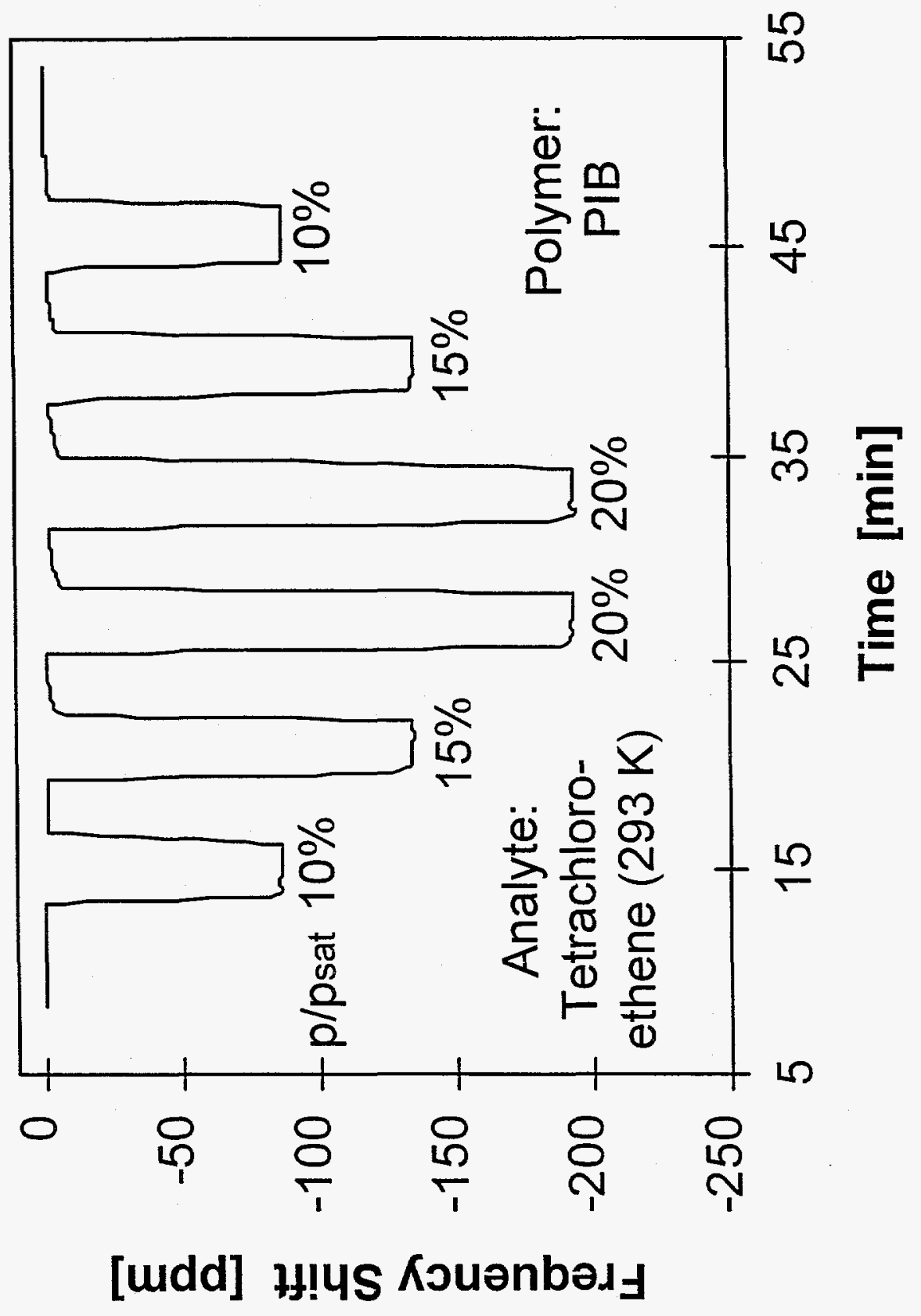


A

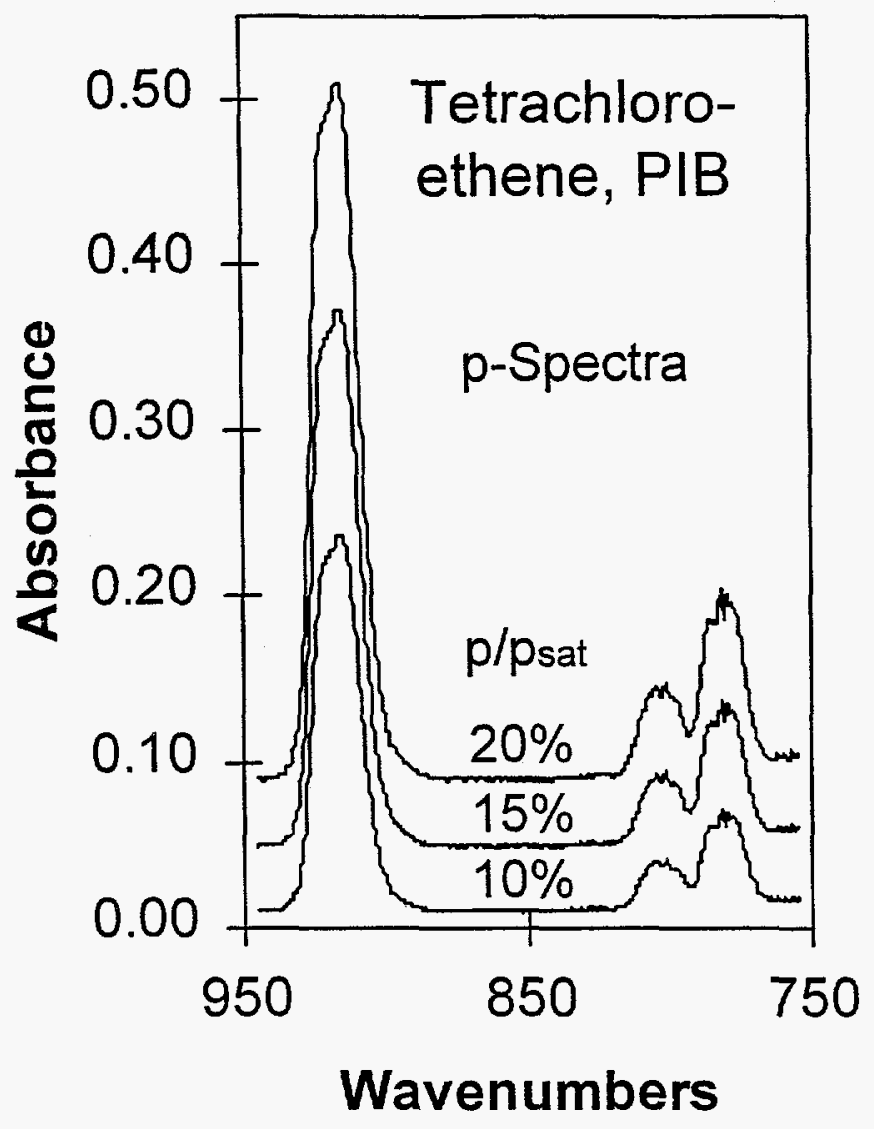

B

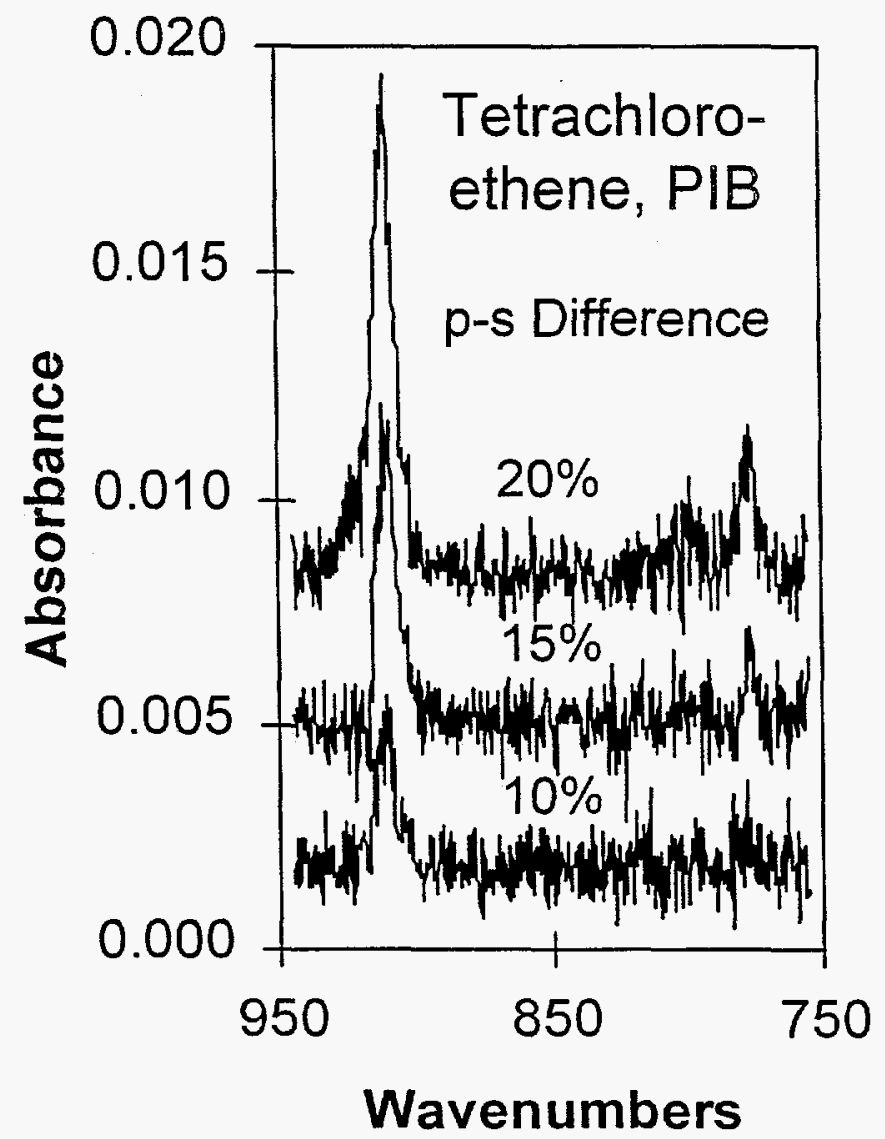

Fig. 3, Thomas et al. 
A

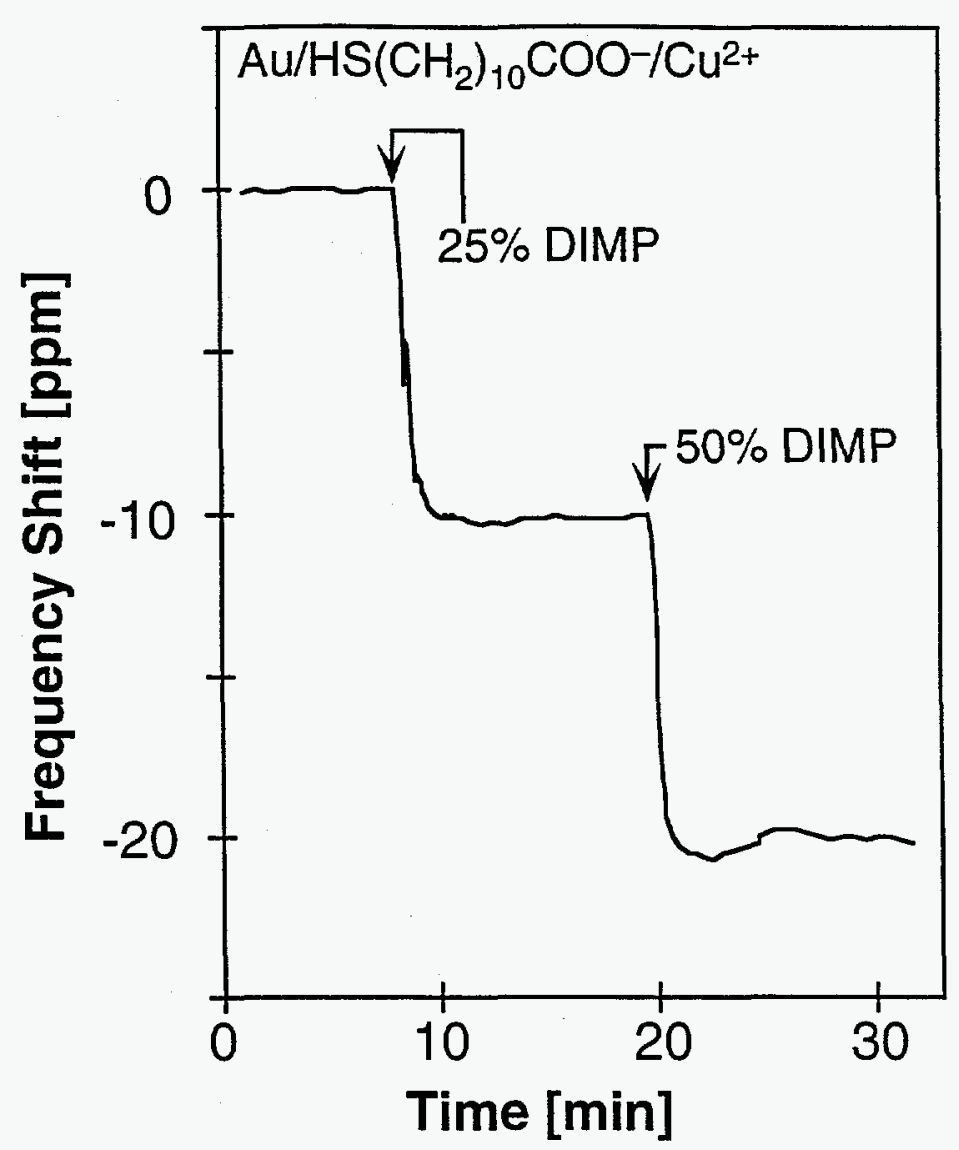

B

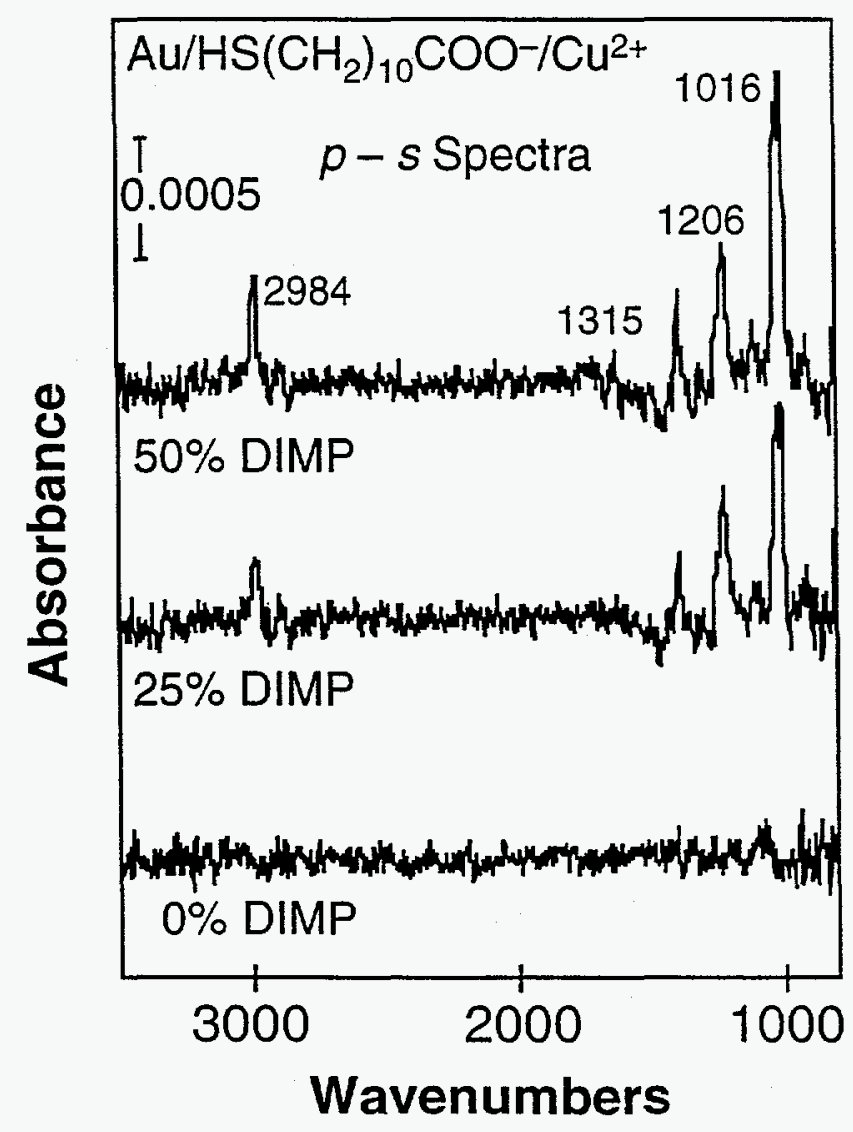

Fig. 4, Thomas et a 
A

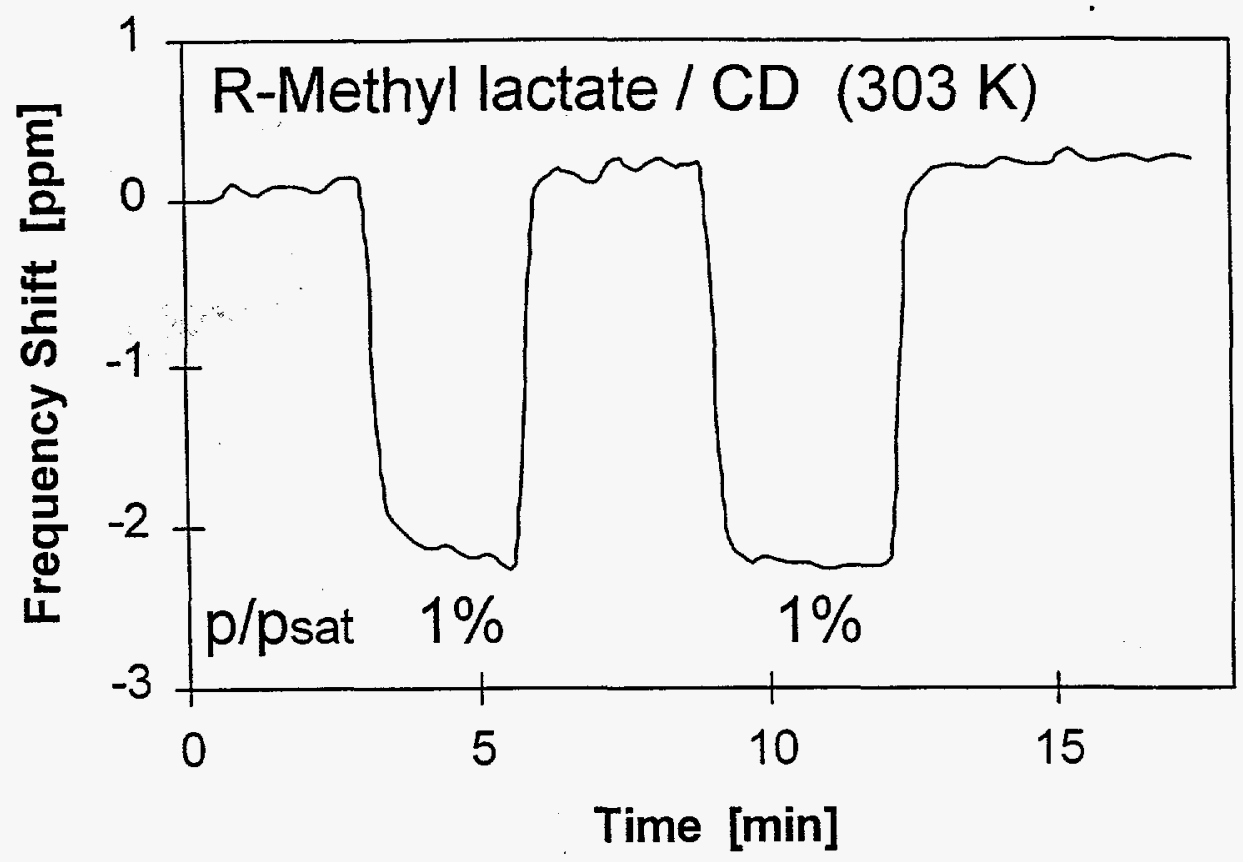

B

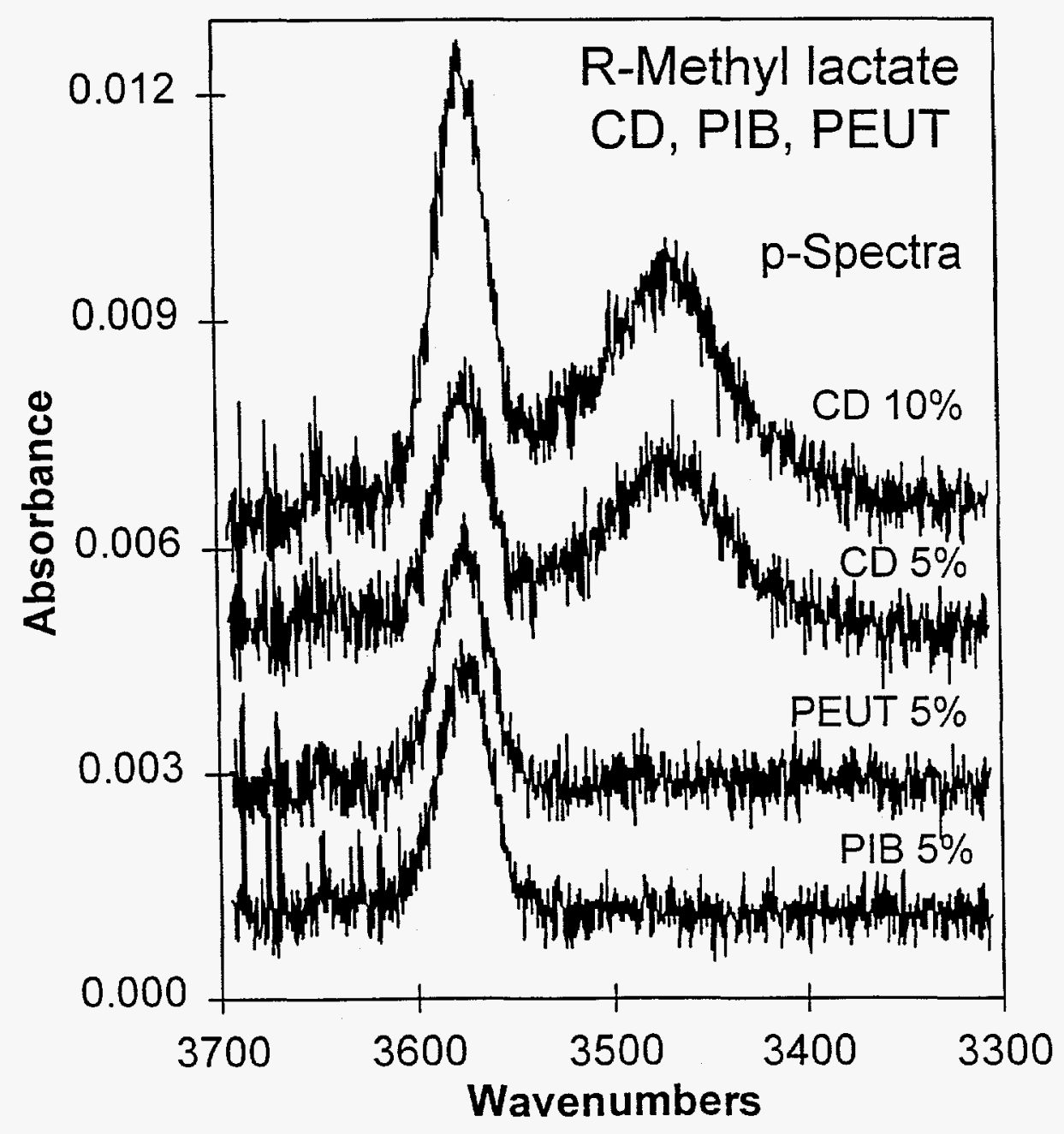

Fig. 5 , Thomas et al. 